\title{
Online-Beobachtung rekombinanter Malariavakzine in einer Multibioreaktoranlage
}

\author{
Kristof Pohlmann ${ }^{1}$, Jens Fricke ${ }^{1}$, Frithjof Tatge ${ }^{1}$, Bart Faber ${ }^{2}$, Reiner Luttmann ${ }^{1}$ \\ 1 HAW - Hochschule für Angewandte Wissenschaften Hamburg \\ 2 BPRC - Biomedical Primate Research Center, Rijswijk, Niederlande
}

\begin{abstract}
Für die Optimierung von Herstellungsprozessen artifizieller Malariavakzine mittels Design of Experiments wurde an der HAW Hamburg eine Multibioreaktoranlage, bestehend aus einem Cellbreeding- und sechs Screeningreaktoren, für einen vollautomatischen Betrieb ausgelegt. Für ein Monitoring des sekretierten Zielproteins wurden zwei unabhängige Messsysteme im Atline-Betrieb an alle sieben Reaktoren angekoppelt. Die Detektion des Zielproteins erfolgt jeweils mit einer Reversed Phase HPLC (RP-HPLC) und in einer Sequentiellen Injektions Analyse mit einer Immobilisierten Metall Ionen Affiniäts Chromatographie (IMAC-SIA). Die sechs Screeningreaktoren der Multibioreaktoranlage werden automatisch sequenziell beprobt. Nach der Auswertung mehrerer Kultivierungen mittels der RP-HPLC konnten aus den Chromatogrammen Informationen zur Online-Beurteilung der Kultivierung und der Produktqualität abgeleitet werden.
\end{abstract}

\section{Herstellung rekombinanter Malariavakzine in einer Multibioreaktor- anlage}

In einem Gemeinschaftsprojekt der HAW und des BPRC zur Herstellung rekombinanter Malariavakzine mit Pichia pastoris wird der Expressionsprozess auf mögliche Einflussfaktoren, wie z. B. pH-Wert, Temperatur, $\mathrm{pO}_{2}$ u.a.m., mittels Design of Experiments (DoE) in einer Multibioreaktoranlage untersucht und optimiert.

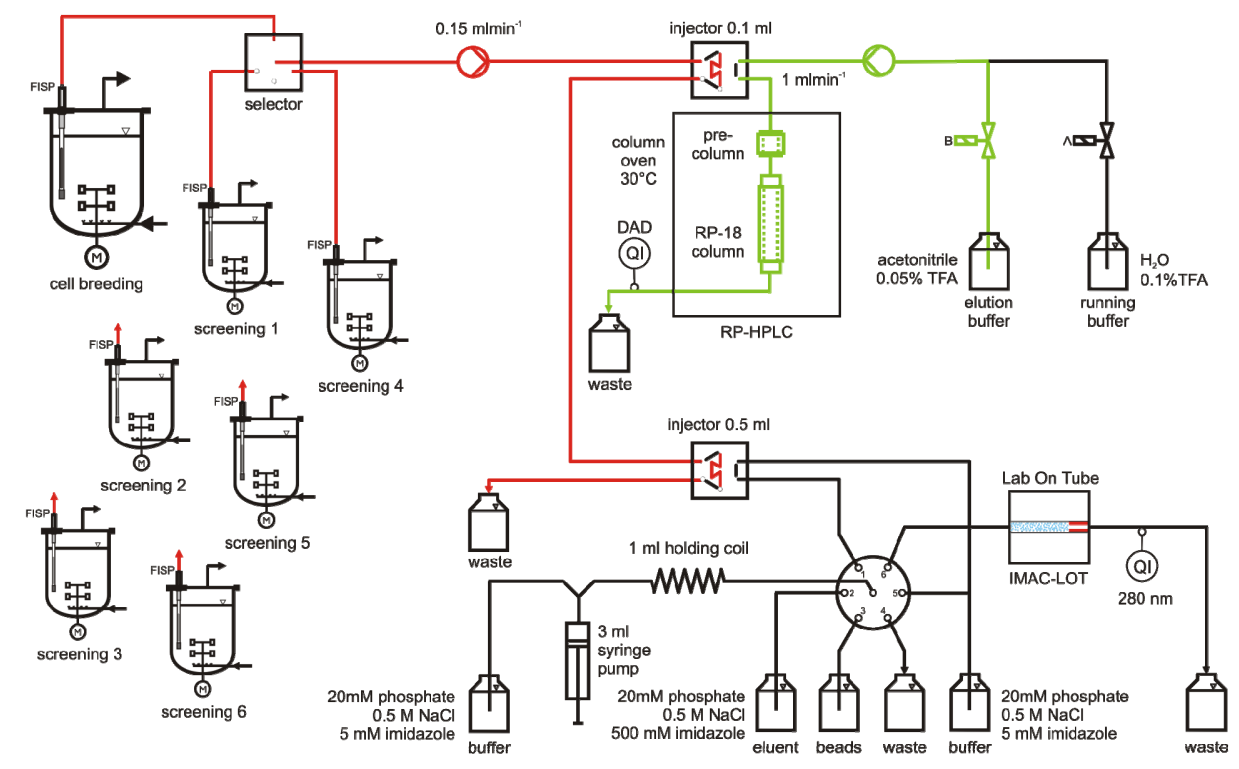

Abbildung 1 Multibioreaktoranlage mit zwei Messsystemen zur Atline-Produktmessung

Hierzu wurden ein BIOSTAT ${ }^{\circledR}$ Bplus Zellanzuchtfermenter und sechs BIOSTAT $^{\circledR}$ Qplus Screeningreaktoren (beide Sartorius Stedim Biotech) prozesstechnisch miteinander verbunden. Die in Abb. 1 schematisch gezeigte Anlage ist für eine parallel/sequenzielle Durchführung von DoEExperimenten konzipiert. [1] 


\section{Ablauf eines Produktionsprozesses}

Die Produktion der Malariavakzine findet in einem dreistufigen Prozess mit einer Batch- und zwei Fed Batch-Phasen zur Produktionsvorbereitung und zur Produktexpression statt. In der BatchPhase wachsen die Zellen $\left(\mathrm{c}_{\mathrm{XL}}\right)$ unlimitiert auf dem Substrat Glycerin, der $\mathrm{pO}_{2}$ fällt bis auf $25 \%$ ab und wird auf diesen Wert geregelt. In der ersten Fed Batch-Phase wird Glycerin limitiert mit einem exponentiellen Profil zugefüttert $\left(\mathrm{Q}_{\mathrm{S} \text { lin }}\right)$. In der Produktionsphase wird die Expression des Zielproteins $\left(\mathrm{c}_{\mathrm{P} 1 \mathrm{M}}\right)$ durch die Zugabe des zweiten Substrates Methanol $\left(\mathrm{c}_{\mathrm{S} 2 \mathrm{M}}\right)$ induziert. Die Methanolkonzentration wird dabei auf einem festgelegten Sollwert gehalten.

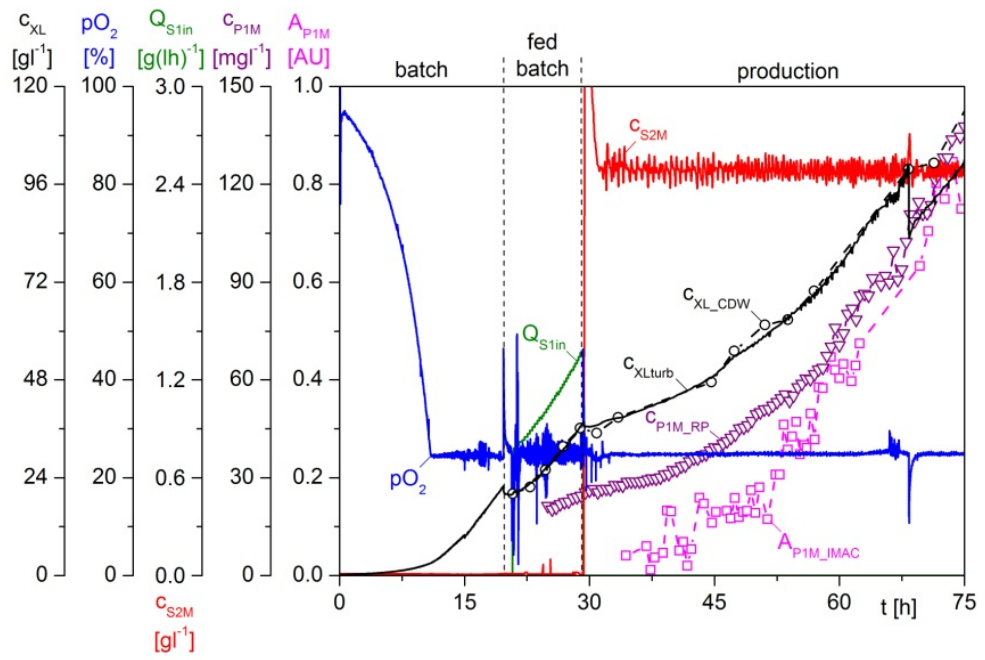

Abbildung 2 Ablauf eines mehrstufigen Produktionsprozesses

Durch die in Abb. 1 gezeigte Messanordnung ist gewährleistet, dass beide Systeme das gleiche Probenmaterial erhalten. Die in Abb. 2 gezeigte Kultivierung zeigt den Prozessablauf in dem die Produktkonzentration mit einer RP-HPLC und einer IMAC-SIA atline gemessen wurde. Die Messergebnisse der SIA weichen bei geringen Konzentrationen stark von den Werten der HPLC ab. In weiteren Versuchen konnte nachgewiesen werden, dass beide Systeme ab einer Zielproteinkonzentration von ca. $50 \mathrm{mgl}^{-1}$ (mit der HPLC gemessen) gut mit einander korrelieren.

\section{Messung des Zielproteins mit einer RP-HPLC und einer IMAC-SIA}

Für die Zielproteinmessung mit einer LaChrom HPLC (VWR International) wird eine LiChrosphere ${ }^{\circledR} 125$ RP-18 Säule verwendet. Grundlage der entwickelten RP-Methode ist eine Gradientenelution aus Wasser mit 0,1 \% TFA als Laufmittel und Acetonitril mit 0,05\% TFA als Elutionsmittel (Abb. 1). Zur Bestimmung der Genauigkeit der Messmethode wurde die Standardabweichung der Einzelmessung für ein Vertrauensintervall von $95 \%$ bestimmt. Diese betrug $3,5 \%$.

In den entsprechenden Chromatogrammen (Abb. 3) konnten vier Peaks nachgewiesen werden, die verschiedene Zielproteinvarianten darstellen. Die Identifikation der Peaks erfolgte mittels SDSPAGE und einem Western Blot mit einem produktspezifischen Antikörper. Durch diese Nachweismethoden lassen sich keine signifikanten Unterschiede zwischen den Peaks feststellen. Daher erfolgte die Bewertung der Proteinkonzentration über die Summe der vier Peakflächen. Diese wurde mittels des Extinktionskoeffizienten in eine Konzentration umgerechnet. Das Verhältnis der einzelnen Peakflächen zu einander variiert im Prozessverlauf und bei veränderten Kultivierungsbedingungen. 

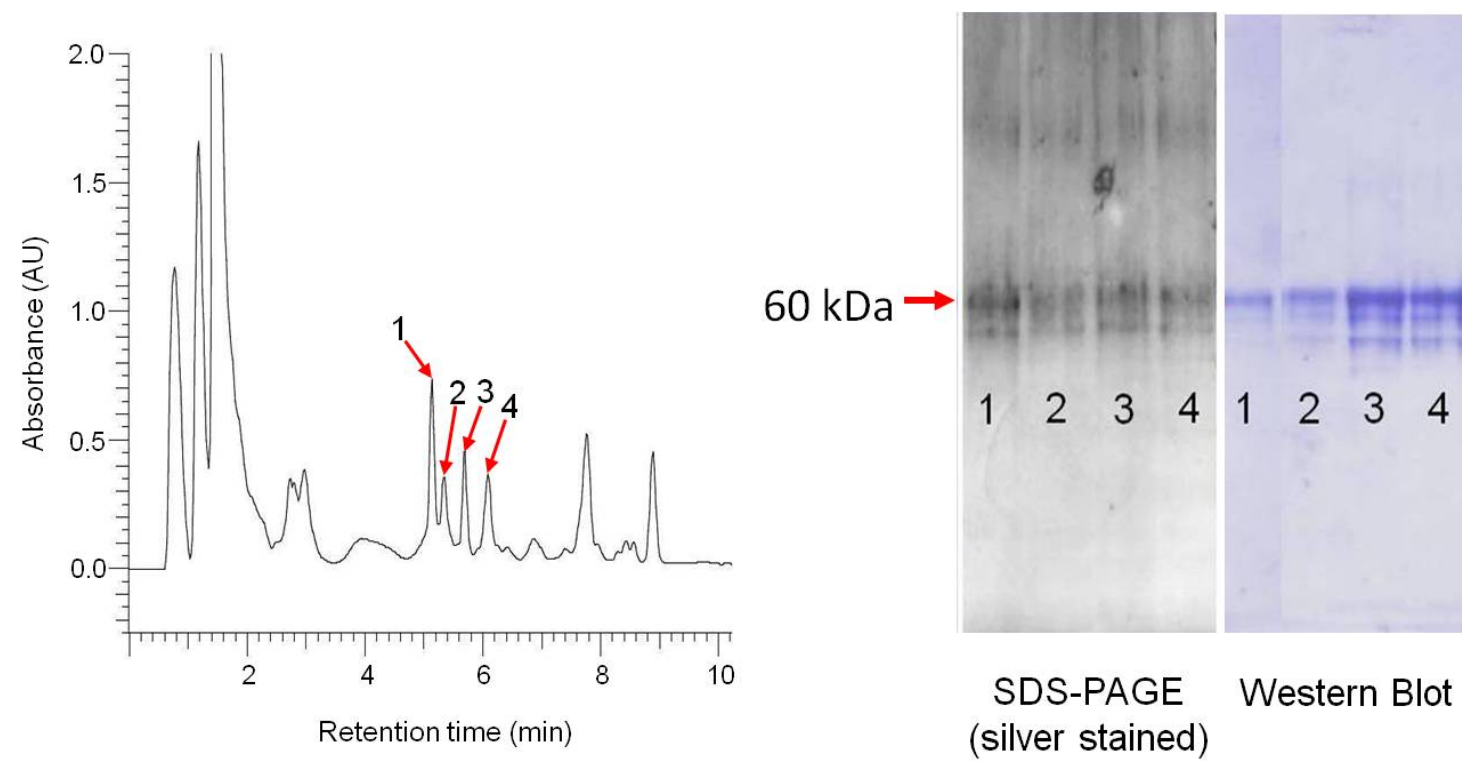

Abbildung 3 Identifikation von Zielproteinpeaks in einer Reversed Phase Chromatographiemethode

Neben der RP-HPLC wurde eine IMAC-Methode zur Messung des Zielproteins etabliert. Bei diesem Verfahren handelt es sich um eine Sequentielle Injektions Analyse (SIA).

Über ein Selektorventil wird Probe aus einer $0,5 \mathrm{~mL}$ fassenden Injektionsschleife mittels einer Spritzenpumpe in die Messkammer gefördert, in der das Zielprotein über die histidinreiche Prosequenz oder einen His-tag immobilisiert wird. Das anschließend mit Imidazol eluierte Zielprotein wird bei $280 \mathrm{~nm}$ in einer Durchflusszelle detektiert (Abb. 1).

\section{Reproduzierbarkeit und Produktqualität}

Bei der Durchführung von DoE-Experimenten sind die Reproduzierbarkeit der Versuche und der Messergebnisse entscheidende Faktoren für die statistische Aussagekraft der Ergebnisse. In den DoE-Experimenten produziert der Zellanzuchtfermenter täglich das Inoculum, das auf die sechs Screeningreaktoren verteilt wird. Dadurch erhalten die Screeningexperimente die gleichen Startbedingungen. Für die Bewertung des DoE werden dann Produktionsläufe mit unterschiedlichen Betriebsparametern durchgeführt.

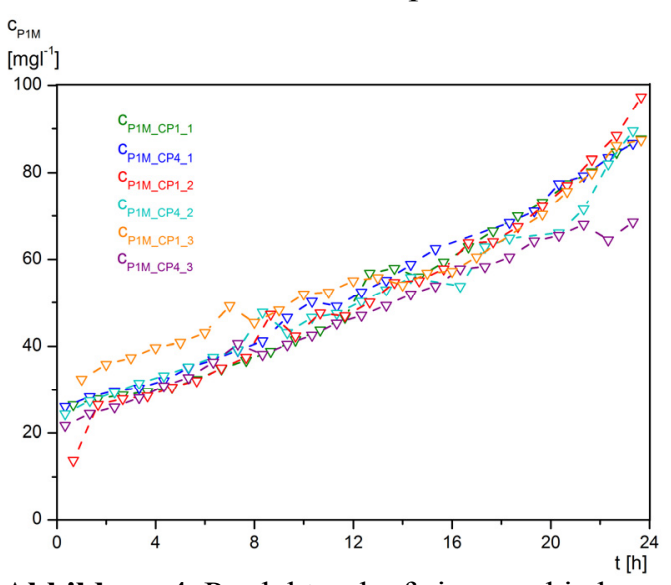

Abbildung 4 Produktverläufe in verschiedenen Centerpoint-Experimenten

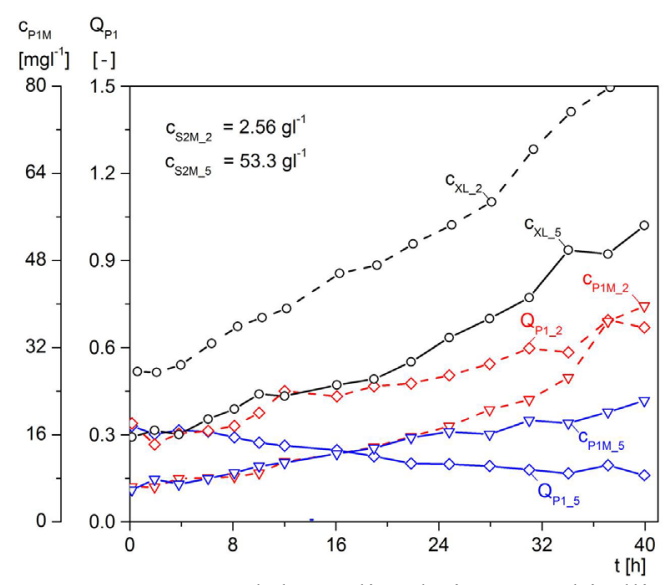

Abbildung 5 Produktqualität bei unterschiedlichen Methanolkonzentrationen 
Um die statistische Varianz der Ergebnisse zu bestimmen, werden Centerpointversuche mit gleichen Betriebsparametern durchgeführt. Eine Bestimmung der Reproduzierbarkeit dieser Experimente erfolgte mittels sechs Kultivierungen, deren Produktmessungen in Abb. 4 dargestellt sind. Alle sechs Versuche zeigen einen vergleichbaren Verlauf hinsichtlich der Expressionsproduktivität.

Durch die Analyse mehrerer dreistufiger Produktionsprozesse konnte auch nachgewiesen werden, dass die Verhältnisse der Proteinvarianten zueinander durch die Kultivierungsbedingungen beeinflussbar sind. Ein besonderes Augenmerk lag hierbei auf Kultivierungen, in denen die Zellen einem künstlichen Kultivierungsstress, z.B. niedrigen und hohen $\mathrm{pH}-$ Werten oder einer sehr hohen Methanolkonzentration im Medium, ausgesetzt waren.

Die Abb. 5 zeigt die Produktkonzentration $c_{P 1 M}(\nabla)$ im Medium, die Zelldichte $c_{X L}(0)$ im Reaktionsraum und die berechnete Produktqualität $\mathrm{Q}_{\mathrm{P} 1}(\diamond)$ von zwei parallel laufenden Versuchen mit unterschiedlichen Animpfkonzentrationen. In dem Bioreaktor Q2 wurde eine für den Produktionsorganismus moderate Methanolkonzentration $\mathrm{c}_{\mathrm{S} 2 \mathrm{M}}$ von 2,56 $\mathrm{gl}^{-1}$ und in dem Bioreaktor Q5 eine Konzentration von 55,3 $\mathrm{gl}^{-1}$ eingestellt.

Die Zelldichten steigen nahezu parallel an, die Produktbildung des Reaktors Q2 ist nach 24 Stunden signifikant höher als die des Reaktors Q5.

Die Produktqualität $\mathrm{Q}_{\mathrm{P} 1}$ zum Messzeitpunkt $\mathrm{t}_{\mathrm{j}}$ in Reaktor Qk,

$$
\mathrm{Q}_{\mathrm{P} 1_{-} \mathrm{k}}\left(\mathrm{t}_{\mathrm{j}}\right)=\frac{\mathrm{IA}_{\mathrm{P} 1 \mathrm{I}_{\mathrm{k}}\left(\mathrm{t}_{\mathrm{j}}\right)}}{\sum_{\mathrm{k}=1}^{4} \mathrm{IA}_{\mathrm{P} 1 \mathrm{i}_{\mathrm{k}} \mathrm{k}}\left(\mathrm{t}_{\mathrm{j}}\right)},
$$

ist definiert als das Verhältnis der Peakfläche des ersten Peaks $\mathrm{IA}_{\mathrm{P} 11}$ zu der Summe aller Peakflächen $\mathrm{IA}_{\mathrm{Pli}}, \mathrm{i}=1$ bis 4. (Abb. 2). Dieses Verhältnis nimmt im Falle des Reaktors Q2 zu, unter den Stressbedingungen im System Q5 hingegen ab. Dieser Trend kann auch bei anderen Kultivierungen beobachtet werden.

\section{Fazit}

Für die Multibioreaktoranlage sind mit der RP-HPLC und der IMAC-SIA zwei robuste und zuverlässige Atline-Messsysteme etabliert worden. Durch die Messergebnisse der RP-HPLC konnte die reproduzierbare Produktion des Malariavakzins in den Screeningreaktoren belegt werden. Dies ist eine Voraussetzung für eine Prozessoptimierung nach DoE. Neben der Produktquantifizierung konnten auch Aussagen bezüglich der Produktqualität und den Auswirkungen von Stress auf den Produktionsorganismus online getroffen werden.

\section{Danksagung}

Die Autoren danken dem Bundesministerium für Bildung und Forschung für die finanzielle Unterstützung des Projektes „Malariavakzine“ (FKZ 1756X09), sowie den Firmen Sartorius Stedim Biotech, BlueSens gas sensor, Stäubli, optek Danulat und MKS Umetrics AB für die Bereitstellung von Geräten und Prozesssoftware. Der für diese Arbeiten verwendete Malariavakzinkandidat wurde unter der Schirmherrschaft der European Malaria Vaccine Development Association entwickelt (Fördernummer LSHP-CT-2007-037506).

\section{Literatur}

[1] J. Fricke, K.Pohlmann, F. Tatge, R. Lang, B. Faber, R. Luttmann: A multi-bioreactor system for optimal production of malaria vaccines with Pichia pastoris: Biotechnol. J. 2011, 6, 437-451 